\title{
Multidisciplinary Treatment of Head and Neck Cancer
}

\author{
Prashanth Varkey, M.Ch., ${ }^{1}$ Yi-Tien Liu, M.D., ${ }^{2}$ and Ngian Chye Tan, M.R.C.S. ${ }^{3}$
}

\section{ABSTRACT}

Head and neck cancer remains a significant cause of morbidity worldwide. Multimodality treatment is often the only way to achieve improved function, quality of life, and survival, calling for a multidisciplinary team approach, particularly in view of the rapid advances being made in various fields. The roles of the head and neck surgeon and reconstructive surgeon are discussed, together with the input afforded by specialists in areas such as diagnostic imaging, radiation therapy, medical oncology, and gene therapy. Telemedicine is of importance in centers where multidisciplinary expertise is not available.

KEYWORDS: Multidisciplinary team, tele-oncology, tumor boards

$\mathrm{H}_{\text {ead }}$ and neck cancer remains a significant cause of morbidity worldwide, with $\sim 400,000$ new cases per year. Multimodality treatment is often the only way to achieve satisfactory results, and this requires clinicians of various fields to come together on a common platform. As science rapidly advances, it would be unwise to expect a single physician to keep abreast of the rapid pace of change in all related fields of cancer treatment. This calls for a multidisciplinary team approach to achieve better treatment results with respect to improved function, quality of life, and survival.

\section{THE MULTIDISCIPLINARY TEAM}

Depending on the resources and the workload at an institution, the members of this team may vary. The broad group of specialties involved include the head and neck surgeon, the reconstructive surgeon, the dental surgeon, and specialists in radiation oncology, medical oncology, medical imaging, clinical pathology and laboratory medicine, pain palliative therapy, nutrition, speech therapy, nursing, and social work. Specialists in newer disciplines such as molecular biology and nuclear imaging are also joining such teams.

\section{THE TUMOR BOARDS}

A hospital tumor board is a multidisciplinary group of physicians who meet on a regular basis to review cancer cases. Through regular meetings, the tumor board will improve the quality of cancer care, provide educational opportunities for participants, and become an asset to the hospital and to the community. Use of multidisciplinary tumor board consultations can ensure that the cancer patient has access to the best current thinking about cancer management. Tumor conferences are an accepted and established institution for the multidisciplinary care of patients with cancer. In our center, evaluation of the patient by the treating physician is followed by a discussion at the weekly tumor conference when the treatment line is decided. This offers the best chance for the patient, as tumor
${ }^{1}$ Division of Reconstructive Microsurgery, Jubilee Mission Hospital, Thrissur, Kerala, India; ${ }^{2}$ Division of Plastic and Reconstructive Surgery, E-Da Hospital, Yanchau, Kaohsiung, Taiwan; ${ }^{3}$ Division of General Surgery, Changi General Hospital, Singapore.

Address for correspondence and reprint requests: Prashanth Varkey, M.Ch., Division of Reconstructive Microsurgery, Jubilee Mission Hospital, Thrissur, Kerala, India 680005 (e-mail: drpvarkey @gmail.com).
Advances in Head and Neck Reconstruction, Part II; Guest Editors, Samir Mardini, M.D., Christopher J. Salgado, M.D., and Hung-Chi Chen, M.D., F.A.C.S.

Semin Plast Surg 2010;24:331-334. Copyright (C) 2010 by Thieme Medical Publishers, Inc., 333 Seventh Avenue, New York, NY 10001, USA. Tel: +1(212) 584-4662.

DOI: http://dx.doi.org/10.1055/s-0030-1263075.

ISSN 1535-2188. 
boards can play a strategic role in the planning of care for cancer patients.

The efficacy of tumor boards was investigated with respect to the recommendations made at tumor conferences and whether these recommendations were enacted. Of the recommendations followed, $16 \%$ were for diagnosis, $78 \%$ were for therapy, and $5 \%$ were for palliation. ${ }^{1}$

\section{THE HEAD AND NECK SURGEON AND THE ROLE OF SURGERY}

The head and neck surgeon is often the torch bearer of the team. Surgery still remains the primary form of treatment of most head and neck cancers. Changes in surgical strategies are resulting in more varied treatments for the same stage of disease with more custom-designed operations for conservation of both form and function. Conservation surgeries are being adopted for laryngeal and thyroid neoplasm. As rightly pointed out by Moore, ${ }^{2}$ conservation surgery in head and neck cancer requires twice as much experience and skill as the so-called radical surgery. Chemotherapy/radiotherapy has come to replace surgery in advanced laryngeal cancer. Although technical advances have been made in microlaryngeal surgery using lasers, a niche still exists for cases that still require conventional surgery. Kehrl and Tolkemitt showed that T2 tumors of the anterior glottic area should preferably be approached by conventional surgery (external approach). ${ }^{3}$ By contrast, T2 tumors of the medial and posterior glottic area should be resected endolaryngeally by laser.

\section{THE RECONSTRUCTIVE SURGEON AND ADVANCES IN HEAD AND NECK RECONSTRUCTION}

A crucial part of head and neck cancer surgery is the reconstruction. This is becoming more challenging as patients demand restoration of form and function as close to normal as possible. More and more flaps are being designed based on a single perforator; this has been made possible due to a better understanding of the perforator anatomy from the studies of Taylor ${ }^{4}$ and others. Several of the perforator flaps combine the advantage of having a more pliable flap as well as decreased donor site morbidity. The ability to raise chimeric flaps ${ }^{5}$ based on a single vascular pedicle allows for optimal usage of tissues in various planes without the need for additional anastomosis.

Despite these advances, certain goals have still not been achieved. These often pertain to form and function. Attempts at functioning muscle transfer for head and neck cancers have not gained popularity as yet. Prefabricated flaps have been used to replace mucosa with mucosa by employing tissue-cultured mucosal sheets
The role of tissue engineering, especially in replacing mucosa, seems promising for the future.

\section{DIAGNOSTIC IMAGING}

The ever-increasing battalion of investigative tools in head and neck cancer has facilitated earlier diagnosis and efficient follow-up. Combined positron emission tomography-computed tomography has gained popularity in many institutions for the initial screening for metastases. Newer advances in this field such as Tc-labeled annexin $\mathrm{V}$ scintigraphy are being investigated. ${ }^{6}$ Lymphoscintigraphy and the role of sentinel node biopsy in mucosal head and neck cancer has been evaluated using multicenter data. The negative predictive value of a negative sentinel node for the remaining neck was $96 \%{ }^{7}$ Use of radiotracer, lymphoscintigraphy, and a handheld gamma probe for lymphatic mapping are the minimal requirements. Use of conventional hematoxylin and eosin staining and immunohistochemistry for cytokeratin is mandatory.

\section{RADIATION THERAPY}

Radiation therapy is an integral arm in the treatment of head and neck cancer and has undergone significant advances. Intensity-modulated radiation therapy (IMRT) and image-guided radiation therapy represent new paradigms in treatment planning and dose delivery. IMRT, an advanced form of external beam irradiation, is a type of three-dimensional conformal radiotherapy (RT). It represents one of the most important technical advances in RT since the advent of the medical linear accelerator. There is significant sparing of critical structures and other normal tissues. IMRT is also capable of delivering different doses to multiple targets, providing a new opportunity for differential dose planning to increase the dose selectively to specific image-defined regions. The technology of RT is clearly undergoing intense and rapid technical developments. It is predicted that radical dose RT will move to proton beam technology and that the treatment will be four-dimensional (the fourth dimension is time). The impact will be higher tumor control probability and reduced frequency and severity of treatment-related morbidity. ${ }^{8}$

\section{MEDICAL ONCOLOGY}

The role of chemotherapy in the treatment of head and neck tumors is unclear. In countries such as India, the proportion of advanced cases is still significantly high, and these patients have dismal survival prospects despite aggressive therapy. Often, surgical resection and/or radiotherapy are not feasible in these patients. Hence, the option of neoadjuvant chemotherapy using effective agents such as ifosfamide and paclitaxel in combination 
with cisplatin was studied and found to have a suitable response. ${ }^{9}$ However, further trials need to be done in this regard.

Chemotherapy has been shown to be most effective when delivered concurrently with radiation for patients with advanced-stage tumors. Concurrent chemoradiation using systemic infusion of cisplatin and 5-fluorouracil in patients with advanced hypopharyngeal cancer has been studied by Endo et al. ${ }^{10}$ Definitive surgery was reserved for residual or recurrent tumors. When concurrent chemoradiation is employed, overall survival is improved, although toxicity can be higher. Studies have also shown a role for concurrent treatment in an effort to avoid total laryngectomy and preserve organ function. ${ }^{11}$

\section{GENE THERAPY}

Advances to conquer malignant tumors have been under development, including gene therapies and molecular target therapy for head and neck cancers. ${ }^{12}$ Tumor cell killing is achieved not by the genes delivered by the vectors, but by the oncolysis induced by the replicated viruses by their original nature. Amplified viral vectors also spread to the adjacent tumor cells and kill these cells in the same manner. Recently, increased evidence for antitumor activity of replication-selective adenoviral vectors has been demonstrated preclinically, and several clinical trials have demonstrated the safety and clinical activity of replication-selective viruses.

\section{VIRTUAL MULTIDISCIPLINARY TEAMS FOR CANCER CARE}

Telemedicine has enabled video-conferencing across long distances, and this has helped establish virtual multidisciplinary teams. Stalfors and Edström et $\mathrm{al}^{13}$ have published their experience with virtual multidisciplinary teams for cancer. At the Amrita Institute of Medical Sciences, Kochi, India, a telemedicine facility has been operative since 2002. This has been able to provide access to multidisciplinary treatment planning for head and neck cancer. The accuracy of tele-oncology has already been studied. In countries with fewer resources to establish many multidisciplinary centers, this may be a promising alternative in the future.

\section{CONCLUSION}

The art of progress is to preserve order amid change and to preserve change amid order.
Change is inevitable. Rapid strides in various disciplines of treatment of head and neck cancer are bound to occur. To keep current with these changes and offer the best of these to the suffering cancer patients necessitates a multidisciplinary team approach. Developing and sustaining such a team assumes a pivotal role in providing quality care in head and neck cancer.

\section{REFERENCES}

1. Petty JK, Vetto JT. Beyond doughnuts: tumor board recommendations influence patient care. J Cancer Educ 2002;17:97-100

2. Moore C. Changing concepts in head and neck surgical oncology: The Hayes Martin Lecture. Am J Surg 1980;140: 480-486

3. Kehrl W, Tolkemitt J, Düsterhus P. [Comparison between transoral microsurgery by $\mathrm{CO} 2$ laser and conventional surgical therapy for T2 glottic carcinoma]. Laryngorhinootologie 2003;82:189-194

4. Taylor GI. The angiosomes of the body and their supply to perforator flaps. Clin Plast Surg 2003;30: 331-342, v

5. Koshima I. A new classification of free combined or connected tissue transfers: introduction to the concept of bridge, siamese, chimeric, mosaic, and chain-circle flaps. Acta Med Okayama 2001;55:329-332

6. van de Wiele C, Lahorte C, Vermeersch H, et al. Quantitative tumor apoptosis imaging using technetium99m-HYNIC annexin V single photon emission computed tomography. J Clin Oncol 2003;21:3483-3487

7. Stoeckli SJ, Pfaltz M, Ross GL, et al. The second international conference on sentinel node biopsy in mucosal head and neck cancer. Ann Surg Oncol 2005;12: 919-924

8. Suit $\mathrm{H}$. [Coming technical advances in radiation oncology]. Z Med Phys 2005;15:215-227

9. Pai VR, Mazumdar AT, Deshmukh CD, et al. Two- vs threedrug combination chemotherapy in advanced or recurrent head and neck cancer: a single institution experience of 361 patients. Med Oncol 2004;21:305-308

10. Endo S, Suzuki S, Tsuji K, et al. [Concurrent chemoradiotherapy for advanced hypopharyngeal cancer. Nippon Jibiinkoka Gakkai Kaiho 2005;108:980-985

11. Devlin J, Sherman E. Combined modality treatment of squamous cell cancer of the head and neck. Clin Adv Hematol Oncol 2005;3:373-382

12. Lamont JP, Kuhn JA, Nemunaitis JJ, McCarty TM. Gene therapy for head and neck cancers. Oncology (Williston Park) 2001;15:303-308; discussion 311-314

13. Stalfors J, Edström S, Björk-Eriksson T, Mercke C, Nyman J, Westin T. Accuracy of tele-oncology compared with face-to-face consultation in head and neck cancer case conferences. J Telemed Telecare 2001;7: 338-343 
